ELECTRONIC RESEARCH ANNOUNCEMENTS OF THE AMERICAN MATHEMATICAL SOCIETY

Volume 5, Pages 18-23 (March 22, 1999)

S $1079-6762(99) 00057-8$

\title{
THE SCHLÄFLI FORMULA IN EINSTEIN MANIFOLDS WITH BOUNDARY
}

\author{
IGOR RIVIN AND JEAN-MARC SCHLENKER
}

(Communicated by Walter Neumann)

\begin{abstract}
We give a smooth analogue of the classical Schläfli formula, relating the variation of the volume bounded by a hypersurface moving in a general Einstein manifold and the integral of the variation of the mean curvature. We extend it to variations of the metric in a Riemannian Einstein manifold with boundary, and apply it to Einstein cone-manifolds, to isometric deformations of Euclidean hypersurfaces, and to the rigidity of Ricci-flat manifolds with umbilic boundaries.

RÉsumé. On donne un analogue régulier de la formule classique de Schläfli, reliant la variation du volume borné par une hypersurface se déplaçant dans une variété d'Einstein à l'intégrale de la variation de la courbure moyenne. Puis nous l'étendons aux variations de la métrique à l'intérieur d'une variété d'Einstein riemannienne à bord. On l'applique aux cone-variétés d'Einstein, aux déformations isométriques d'hypersurfaces de $E^{n}$, et à la rigidité des variétés Ricci-plates à bord ombilique.
\end{abstract}

Let $M$ be a Riemannian $(m+1)$-dimensional space-form of constant curvature $K$, and $\left(P_{t}\right)_{t \in[0,1]}$ a one-parameter family of polyhedra in $M$ bounding compact domains, all having the same combinatorics. Call $V_{t}$ the volume bounded by $P_{t}$, $\theta_{i, t}$ and $W_{i, t}$ the dihedral angle and the $(m-1)$-volume of the codimension 2 face $i$ of $P_{t}$. The classical Schläfli formula (see [Mil94] or [Vin93]) is

$$
\sum_{i} W_{i, t} \frac{d \theta_{i, t}}{d t}=m K \frac{d V_{t}}{d t}
$$

This formula has been extended and used on several occasions recently; see for instance [Hod86], [Bon].

We give a smooth version of this formula, for 1-parameter families of hypersurfaces in (Riemannian of Lorentzian) Einstein manifolds. Then we extend it to variations of an Einstein metric inside a manifold with boundary (a much more general process in dimension above 3). Finally, we give three applications: to the variation of the volume of Einstein cone-manifolds, to isometric deformations of hypersurfaces in the Euclidean space, and to the rigidity of Ricci-flat manifolds with umbilic boundaries. The reader can find the details in [RS98].

Throughout this paper, $M$ is an Einstein manifold of dimension $m+1 \geq 3$, and $D$ is its Levi-Civita connection. When dealing with a hypersurface $\Sigma$ (resp. with

Received by the editors July 31, 1998.

1991 Mathematics Subject Classification. Primary 53C21; Secondary 53C25.

Key words and phrases. Vanishing theorems; null spaces.

(C)1999 American Mathematical Society 
the boundary $\partial M$ ), we call $I$ the induced metric, also called the first fundamental form, of the corresponding immersion in $M . \bar{D}$ is the Levi-Civita connection of $I$, $B$ the shape operator, and $I I, I I I$ are the second and third fundamental forms of $\Sigma$; $B$ and $I I$ are defined with respect to an oriented unit normal vector to $\Sigma$ (resp. to the exterior unit normal to $\partial M$ ).

The trace $H$ of $B$ is called the "mean curvature" (some definitions differ by a factor $m$ ) and the "higher mean curvatures" $H_{k}, k \geq 1$, are the higher symmetric functions of the principal curvatures of $\Sigma$ (resp. $\partial M)$. For instance, $H_{2}=\left(H^{2}-\right.$ $\left.\operatorname{tr}\left(B^{2}\right)\right) / 2 . d V, d A$ are the volume elements in $M$ and on $\Sigma$ (resp. $\partial M$ ) respectively.

We denote by $\delta$ the divergence acting on symmetric tensors, and by $\delta^{*}$ its formal adjoint. We will often implicitly identify (through the metric) vector fields and 1 -forms, as well as quadratic forms and linear morphisms.

\section{Deformation of hypersurfaces}

Here is an analogue of the Schläfli formula for deformations of (smooth) hypersurfaces in a fixed Einstein manifold $M$, which can be Riemannian or Lorentzian (the other pseudo-Riemannian cases can be treated in the same way).

Theorem 1. Let $\Sigma$ be a smooth oriented hypersurface in a (Riemannian) Einstein $(m+1)$-manifold $M$ with scalar curvature $S$, and $v$ a section of the restriction of $T M$ to $\Sigma$. $v$ defines a deformation of $\Sigma$ in $M$, which induces variations $V^{\prime}, H^{\prime}$ and $I^{\prime}$ of the volume bounded by $\Sigma$, mean curvature, and induced metric on $\Sigma$. Then:

$$
\frac{S}{m+1} V^{\prime}=\int_{\Sigma}\left(H^{\prime}+\frac{1}{2}\left\langle I^{\prime}, I I\right\rangle\right) d A
$$

The unit normal used to define $H$ and II should be toward the outside of the volume bounded by $\Sigma$. Actually $\Sigma$ does not need to bound a finite volume domain for this formula to hold. Otherwise, $V^{\prime}$ is just the derivative of the (signed) volume contained between $\Sigma$ and $\Sigma_{t}$, for small $t$. This volume is then oriented by the unit normal to $\Sigma_{t}$ used to define $H$ and $I$, which should be toward the exterior.

The proof can be given separately for normal and for tangent deformations of $\Sigma$. For tangent deformations, $V^{\prime}=0$, and the relation between $I^{\prime}$ and $H^{\prime}$ comes from the equation: $\delta I I=-d H$, which holds because $M$ is Einstein. For normal deformations, $H^{\prime}$ is obtained using the trace of $I^{\prime}$, which is related to the Laplacian of the amplitude of the deformation and to the Ricci curvature of $M$ on the normal to $\Sigma$.

The "classical" Schläfli formula (1) for polyhedra in space-forms follows from Theorem 1. Namely, one can apply Theorem 1 to the set of points at distance $r$ on the outside of a convex polyhedron and let $r \rightarrow 0$.

In the Lorentzian case, the only difference is that now $g(n, n)=-1$, so the volume variation has a minus sign in the formula. Applying the Lorentzian formula to the set of points at distance $\epsilon$ from a polyhedron in $S_{1}^{n}$, one obtains the Schläfli formula for de Sitter polyhedra as in [SP97].

\section{EINSTEIN MANIFOLDS WITH BOUNDARY}

Here $(M, \partial M)$ is a compact manifold with boundary with an Einstein metric $g$ of scalar curvature $S$. We will prove the same formula as in the previous section, but in a much more general setting: instead of moving a hypersurface in an Einstein manifold, we will be changing the metric (among Einstein metrics of a given scalar 
curvature) inside this manifold with boundary. Although the two operations are equivalent in dimension at most 3 , moving the inside metric is much more general in higher dimensions. On the other hand, our proof only works for Riemannian Einstein manifolds. It is not obvious whether it can be extended to the pseudoRiemannian setting.

If $g$ is an Einstein metric, we say that a 2-tensor $h$ is an "Einstein variation" of $g$ if the associated variation of the metric induces a variation of the Ricci tensor which is proportional to $h$, so that $g+\epsilon h$ remains, to the first order, an Einstein manifold with constant scalar curvature.

Theorem 2. Let $h$ be a smooth Einstein variation of $g$. Then:

$$
\frac{S}{m+1} V^{\prime}=\int_{\partial M} H^{\prime}+\frac{1}{2}\left\langle h_{\mid \partial M}, I I\right\rangle d A .
$$

As always when studying deformations of Riemannian metrics, the proof needs put some kind of restriction to remove the geometrically trivial deformations, which only correspond to the action of vector fields on the metric. We prevent those deformations in the same way as e.g. in [GL91], [DeT81] or [Biq97], that is, we only consider metric variations $h$ such that $2 \delta h+d \operatorname{tr} h=0$. The following proposition, which is proved by a fairly simple variational argument, shows that we do not forget any metric variation when doing this.

Proposition 1. Let $h^{\prime}$ be a smooth variation of $g$. Suppose that either $S \leq 0$, or $M$ is strictly convex. There exists another smooth variation $h$ of $g$ such that $2 \delta h+d \operatorname{tr}(h)=0$ and $h=h^{\prime}+\delta^{*} v_{0}$, where $v_{0}$ is a vector field vanishing on $\partial M$.

The variation $h$ of $g$ obtained in Proposition 1 satisfies (because the metric remains Einstein) a simple, elliptic equation. Taking its trace shows that the trace of $h$ also satisfies an elliptic equation which, when integrated over $M$, expresses $V^{\prime}$ as some integral over $\partial M$. A careful examination of this boundary term leads to Theorem 2.

\section{Applications}

A first application can be found by looking at "singular objects", just as to go from the smooth Theorem 1 to the classical Schläfli formula. There are no polyhedra in general Einstein manifolds, but we can check what happens when we deform Einstein manifolds with cone singularities. It should be pointed out that some of the most interesting modern uses of the classical Schläfli formula concern hyperbolic 3 -dimensional cone-manifolds.

Let $M$ be a compact $(m+1)$-manifold, and $N$ a compact codimension 2 submanifold of $M$. Suppose $\left(g_{t}\right)$ is a 1-parameter family of Einstein metrics with fixed scalar curvature $S \leq 0$ on $M \backslash N$, with a conical singularity on $N$ in the sense that, in normal coordinates around $N, g_{t}$ has an expansion like

$$
g_{t}=h_{t}+d r^{2}+r^{2} d \theta^{2}+o\left(r^{2}\right),
$$

where $h_{t}$ is the metric induced on $N$ by $g_{t}$, and $\theta \in \mathbf{R} / \alpha_{t} \mathbf{Z}$ for some $\alpha_{t} \in \mathbf{R}$. Call $V_{t}$ the volume of $\left(M \backslash N, g_{t}\right)$, and $W_{t}$ the volume of $\left(N, h_{t}\right)$. Then

Corollary 1. $V_{t}$ varies as: $\frac{S}{m+1} \frac{d V_{t}}{d t}=W_{t} \frac{d \alpha_{t}}{d t}$.

Note. The same result holds when $N$ has several connected components, each with a different value of $\alpha_{t}$. N could also be replaced by a stratified submanifold. 
Example. Take $m+1=3$ in the previous example. We find the Schläfli formula for the variation of the volume of a hyperbolic cone-manifold [Hod86].

When the $W_{i, t}$ are constant, the left-hand side of (1) is a polyhedral analogue of the variation of the mean curvature integral of a hypersurface. When $K=0$, the right-hand side is 0 . This shows that the "mean curvature" of a 1-parameter family of Euclidean polyhedra with constant induced metric is constant. This was used in [AR97] to prove, using geometric measure theory methods, the following

Theorem 3. In $\mathbf{R}^{m+1}$, the integral of the mean curvature remains constant in an isometric deformation of a hypersurface.

This theorem follows immediately from our Theorem 1 .

On the other hand, the integral mean curvature is not determined by the metric on $\partial M$ : this is already visible in $\mathbf{R}^{3}$. Namely, some metrics on $S^{2}$ admit two isometric embeddings in $\mathbf{R}^{3}$ : the classical example is that a (topological) sphere in $\mathbf{R}^{3}$ which is tangent to a plane along a circle can be "flipped" so as to obtain another embedding with the same induced metric [Spi75]. Those two embeddings do not in general have the same integral mean curvature - and thus we have a complicated way of seeing that the two flipped surfaces cannot be bent one into the other.

Formula (3) is even simpler for variations which vanish on $\partial M$ :

Theorem 4. If $h$ is a smooth Einstein variation of $g$ which does not change the induced metric on $\partial M$, then

$$
\int_{\partial M} H^{\prime} d V=\frac{S}{m+1} V^{\prime}
$$

In particular, for $S=0$, this implies that the integral of the mean curvature of the boundary is constant under an Einstein variation which does not change the induced metric on $\partial M$; this is a direct generalization of Theorem 3 .

The analogue of Theorem 3 is also true, but in a pointwise sense, for the higher mean curvatures:

Theorem 5. In $\mathbf{R}^{m+1}$, the integral of $H_{k}(k \geq 2)$ remains constant in an isometric deformation of a hypersurface.

This comes from the following (probably classical) description of the possible isometric deformations of a hypersurface for $m+1 \geq 4$ :

Remark 1. Let $\left(\Sigma_{t}\right)_{t \in[0,1]}$ be a 1-parameter family of hypersurfaces in a space-form, such that the induced metric $I_{t}$ is constant to the first order at $t=0$. Then, at each point, one of the following is true:

- $I_{0}=0$

- $\operatorname{rk}\left(I_{0}\right) \leq 2$, and $I_{0}^{\prime}$ vanishes on the kernel of $I_{0}$;

- $I_{0}^{\prime}=0$;

where $I_{t}$ is the second fundamental form of $\Sigma_{t}$, and $I_{t}^{\prime}$ its variation.

Theorem 5 clearly follows, because $H_{k}^{\prime}$ is zero for $k \geq 3$ in each case, and the Gauss formula gives the proof for $k=2$.

Denote by $\Sigma_{t}^{\epsilon}$ the parallel surface at distance $\epsilon$ from $\Sigma_{t}$. It is well known (see, e.g., Santalo's book [San76]) that the area of $\Sigma^{\epsilon}$ is a polynomial in $\epsilon$ where the coefficent of $\epsilon^{k}$ is (essentially) the $k$-th mean curvature of $\Sigma$. The two Theorems 3 and 5 can then be combined as stating that: 
Theorem 6. The area of $\Sigma_{t}^{\epsilon}$ stays constant when $\Sigma_{t}$ is a bending of $\Sigma_{0}$.

Finally, we use the Schläfli formula above to prove a rigidity result for Ricciflat manifolds with umbilic boundary; it is a generalization of the classical result (see [Spi75]) that the round sphere is rigid in $\mathbf{R}^{3}$, that is, it cannot be deformed smoothly without changing its induced metric.

Lemma 1. Suppose $(M, \partial M)$ is a compact $(m+1)$-manifold with boundary, and $\left(h_{t}\right)_{t \in[0,1]}$ is a nontrivial 1-parameter family of Ricci-flat metrics on $M$ inducing the same metric on $\partial M$, and such that $\partial M$ is umbilic and convex (or concave) for $h_{0}$. Then $\partial M$ has at least two connected components, and $\left(h_{t}\right)$ corresponds to the displacement of some connected component(s) of $\partial M$ under the flow of some Killing field(s) of $M$.

This kind of rigidity result could be used in the future to prove that, given a Ricciflat manifold $M$ with umbilic boundary and induced metric $g_{0}$ on the boundary, any metric close to $g_{0}$ on $\partial M$ can be realized as induced on $\partial M$ by some Ricci-flat metric on $M$. In this setting, rigidity corresponds to the local injectivity of an operator sending the metrics on $M$ to the metrics on $\partial M$. In dimension 3 , this would be a part of the classical result (see [Nir53]) that metrics with curvature $K>0$ on $S^{2}$ can be realized as induced by immersions into $\mathbf{R}^{3}$. This circle of ideas is illustrated in [Sch98].

The first point is to understand what an umbilic hypersurface in an Einstein manifold is. By definition, if $N$ is a Riemannian manifold and $S$ is a hypersurface, then $S$ is umbilic if, at each point $s \in S$, II is proportional to $I$, with a proportionality constant $\lambda(s)$ depending on $s$. Now

Remark 2. If $N$ is Einstein, then $\lambda$ is constant on each connected component of $S$.

The next step is an inequality concerning the integral of the mean curvature squared.

Proposition 2. Let $(M, g)$ be an Einstein manifold with boundary, with scalar curvature $S$. Call $\bar{S}$ the scalar curvature of $\left(\partial M, g_{\mid \partial M}\right)$. Then the mean curvature $H=\operatorname{tr}(I)$ of $\partial M$ satisfies

$$
\frac{\bar{S}}{m-1}-\frac{S}{m+1} \leq \frac{H^{2}}{m}
$$

with equality if and only if $\partial M$ is umbilic.

Proposition 2 and Theorem 4 lead to the proof of Lemma 1. The key point is that by Proposition $2, H^{2}$ is pointwise minimal when the boundary is umbilic, while Theorem 4 shows that the integral of $H$ is constant. The boundary therefore has to remain umbilic in an Einstein variation which vanishes on the boundary.

\section{ACKNOWLEDGEMENTS}

The authors are happy to acknowledge their debt to Fred Almgren, without whom this paper would not have been possible. I. Rivin would like to thank the Institut Henri Poincaré and the Institut des Hautes Études Scientifiques for their hospitality at crucial moments. 


\section{REFERENCES}

[AR97] F. O. Almgren and I. Rivin. The mean curvature integral is invariant under bending. Manuscript, 1997.

[BG93] M. Berger and B. Gostiaux. Géométrie différentielle: variétés, courbes et surfaces. Presses universitaires de France, 1993. MR 93j:53001

[Biq97] O. Biquard. Métriques d'Einstein asymptotiquement symétriques. Prépublication no. 9724, Ecole Polytechnique, 1997.

[Ble96] David D. Bleecker. Volume increasing isometric deformation of convex polyhedra. Journal of Differential Geometry, 43:505-526, 1996. MR 97g:52035

[Bon] F. Bonahon. A Schläfli-type formula for convex cores of hyperbolic 3-manifolds. Journal of Diff. Geometry. To appear.

[Con77] R. Connelly. A counterexample to the rigidity conjecture for polyhedra. Inst. Haut. Etud. Sci., Publ. Math., 47:333-338, 1977. MR 58:7642

[DeT81] D. M. DeTurck. Existence of metrics with prescribed Ricci curvature: local theory. Invent. Math., 65:179-207, 1981. MR 83b:53019

[GL91] C. R. Graham and J. M. Lee. Einstein metrics with prescribed conformal infinity on the ball. Adv. Math., 87:186-225, 1991. MR 92i:53041

[Her79] G. Herglotz. Gesammelte Schriften. Herausgegeben im Auftrage der Akademie der Wissenschaften in Goettingen von Hans Schwerdtfeger. Vandenhoeck \& Ruprecht, 1979. MR 80h:01040

[Hod86] C. Hodgson. PhD Thesis, Princeton University, 1986.

[Lie00] H. Liebmann. Ueber die Verbiegung der geschlossenen Fläschen positiver Krümmung. Math. Annalen, 53:81-112, 1900.

[Mil94] J. Milnor. The Schläfli differential equality. In Collected papers, vol. 1. Publish or Perish, 1994. MR 95c:01043

[Nir53] L. Nirenberg. The Weyl and Minkowski problems in differential geometry in the large. Comm. Pure Appl. Math, 6:337-394, 1953. MR 15:347b

[Pog73] A. V. Pogorelov. Extrinsic Geometry of Convex Surfaces. American Mathematical Society, 1973. Translations of Mathematical Monographs. Vol. 35. MR 49:11439

[RS98] I. Rivin and J-M. Schlenker. Schläfli formula for Einstein manifolds. IHES Preprint, 1998.

[San76] L. Santaló. Integral geometry and geometric measure theory. Addison-Wesley, Reading, MA, 1976. MR 55:6340

[Sch98] J.-M. Schlenker. Einstein manifolds with convex boundaries. Prépublication no. 98-12, Université de Paris-Sud, 1998.

[SP97] E. Suarez-Peiró. A Schläfli formula for simplices in semi-Riemannian hyperquadrics, Gauss-Bonnet formulas for simplices in the de Sitter sphere and the dual volume of a hyperbolic simplex. Manuscript, 1997.

[Spi75] M. Spivak. A comprehensive introduction to geometry, Vols. I-V. Publish or perish, 1970-1975. MR 42:2369; MR 42:6726; MR 51:8962; MR 52:15254a; MR 52:15254b

[Vin93] D. V. Alekseevskij, E. B. Vinberg, and A. S. Solodovnikov. Geometry of Spaces of Constant Curvature, Encyclopaedia of Math. Sci., 29, Springer, Berlin, 1993. MR 95b:53042

Department of Mathematics, University of Manchester, Oxford Road, Manchester M13 9PL, G.B.

E-mail address: irivin@ma.man.ac.uk

Topologie et Dynamique (URA 1169 CNRS), BÂt. 425, Université De Paris-Sud, 91405 Orsay Cedex, France

E-mail address: jean-marc.schlenker@math.u-psud.fr 